\title{
Baikal Neutrino Telescope - an underwater laboratory for astroparticle physics and environmental studies
}

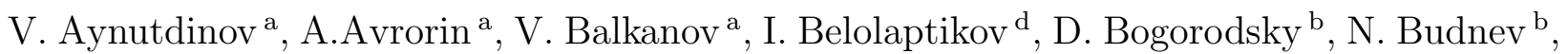
I. Danilchenko ${ }^{a}$, G. Domogatsky ${ }^{a}$, A. Doroshenko ${ }^{a}$, A. Dyachok ${ }^{b}, Z_{\text {Zh.-A. Dzhilkibaev }}{ }^{\text {a }}$ S. Fialkovsky ${ }^{\text {f }}$, O. Gaponenko ${ }^{\text {a }}$, K.Golubkov ${ }^{\text {d }}$, O. Gress ${ }^{\text {b }}$, T. Gress ${ }^{\text {b }}$, O. Grishin ${ }^{\text {b }}$, A. Klabukov ${ }^{\text {a }}$, A. Klimov ${ }^{\text {h }}$, A.Kochanov ${ }^{\text {b }}$, K. Konischev ${ }^{d}$, A. Koshechkin ${ }^{a}$, V. Kulepov ${ }^{f}$, L. Kuzmichev ${ }^{\text {c }}$, S. Lovtsov ${ }^{\text {b }}$, E. Middell ${ }^{\text {e }}$, S. Mikheyev ${ }^{\mathrm{a}}$, M. Milenin ${ }^{\mathrm{f}}, \mathrm{R}$. Mirgazov ${ }^{\mathrm{b}}$, E. Osipova ${ }^{\mathrm{c}}$, G. Pan'kov $^{\mathrm{b}}$, L. Pan'kov $^{\mathrm{b}}$, A. Panfilov ${ }^{\mathrm{a}}$, D. Petukhov ${ }^{\mathrm{a}}$, E. Pliskovsky ${ }^{\mathrm{d}}$, P. Pokhil $^{\mathrm{a}}$, V. Poleschuk ${ }^{\mathrm{a}}$, E. Popova $^{\mathrm{c}}$, A. Rastegin $^{\mathrm{b}}$, V. Prosin ${ }^{c}$, M. Rozanov ${ }^{g}$, V. Rubtzov ${ }^{b}$, A. Sheifler ${ }^{\mathrm{a}}$, A. Shirokov ${ }^{\mathrm{c}}$, B. Shoibonov ${ }^{\mathrm{d}}$, Ch. Spiering ${ }^{\mathrm{e}}$, B. Tarashansky ${ }^{\mathrm{b}}, \mathrm{R}$. Wischnewski ${ }^{\mathrm{e}}, \mathrm{I}_{\text {. Yashin }}{ }^{\mathrm{c}}, \mathrm{V}$. Zhukov ${ }^{\mathrm{a}}$

${ }^{\mathrm{a}}$ Institute for Nuclear Research, 60th October Anniversary pr. 7a, Moscow 117312, Russia

b Irkutsk State University, Irkutsk, Russia

${ }^{\mathrm{c}}$ Skobeltsyn Institute of Nuclear Physics MSU, Moscow, Russia

d Joint Institute for Nuclear Research, Dubna, Russia

e DESY, Zeuthen, Germany

${ }^{\mathrm{f}}$ Nizhni Novgorod State Technical University, Nizhni Novgorod, Russia

g St.Petersburg State Marine University, St.Petersburg, Russia

${ }^{\mathrm{h}}$ Kurchatov Institute, Moscow, Russia

\begin{abstract}
We review the status of the Baikal Neutrino Experiment. The Neutrino Telescope NT200 is operating in Lake Baikal since 1998 and has been upgraded to the 10 Mton detector NT200+ in 2005. We present selected results concerning astroparticle physics as well as results of environmental studies. We describe the strategy of creating a Gigaton (km3) scale neutrino detector at Lake Baikal. First steps of activities towards a km3 Baikal neutrino telescope, including the development of acoustic high energy neutrino detection method are discussed.
\end{abstract}

Key words: Neutrino telescopes, Neutrino astronomy, UHE neutrinos, BAIKAL PACS: 95.55.Vj, 95.85.Ry, 96.40.Tv

\section{Introduction}

Lake Baikal in Siberia is one of the most extraordinary lakes in the world. It is the world's largest reservoir of liquid fresh surface water, and it is a home to several hundred of endemic species as well as to the first underwater Cherenkov high energy neutrino detector. The Baikal Neutrino Telescope NT200 takes data since April 1998. On April 9 ${ }^{\text {th }}$, 2005, the 10-Mton scale detector NT200+ was put into operation in Lake Baikal. Description of detector configuration and performance have been given elsewhere [1-5]. Next milestone of the ongoing km3-telescope research and development work (R\&D) was the installation of a "new technology" prototype string as a part of NT200+ in spring 2008. As well, during some years, we have been studying a possibility of acoustic detection of ultra-high energy neutrinos in Lake Baikal. In this paper we review the current status of the Baikal Neutrino Experiment [7], the activities toward the km3-scale detector [6] and acoustic detection of super high energy neutrinos.

The progress of the Baikal Neutrino Experiment is connected, to a considerable degree, with the favorable natural conditions of the site - thus the site study is an important part of the project. At the moment, the list of important for Lake Baikal ecosystem problems, that was studied, is rather extensively due to the use of new technologies, instruments and methods designed in the framework of the experiment. In the end of the paper we will discuss briefly some results of the site studies.

\section{Selected results obtained with NT200}

\subsection{Atmospheric neutrinos}

The signature of charged current muon neutrino events is a muon crossing the detector from below. Muon track reconstruction algorithms and background rejection have 
been described elsewhere [8]. Compared to [8] the analysis of the 4-year sample (1038 days live time) was optimized for higher signal passing rate, and accepting a slightly higher contamination of $15-20 \%$ fake events [9]. A total of 372 upward going neutrino candidates were selected. From Monte-Carlo simulation a total of 385 atmospheric neutrino and background events are expected. The skyplot of these events is shown in Fig. 1.

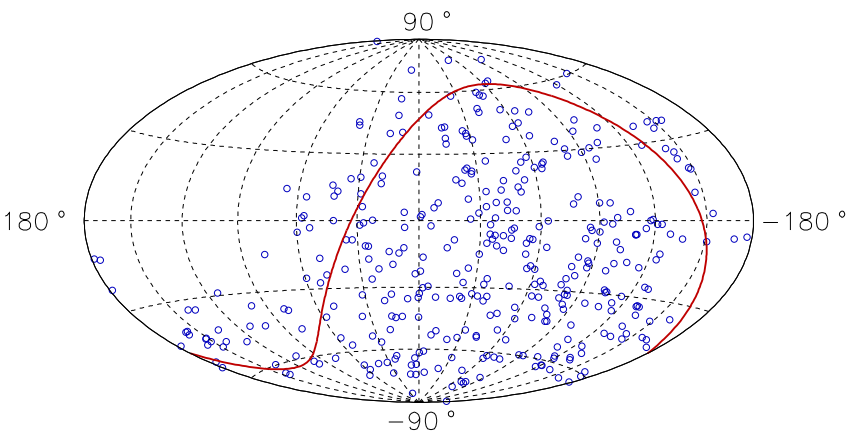

Fig. 1. Skyplot (galactic coordinates) of neutrino events for five years. The solid curve shows the equator.

\subsection{Search for Neutrinos from WIMP Annihilation}

The search for WIMPs with the Baikal neutrino telescope is based on a possible signal of nearly vertically upward going muons, exceeding the flux of atmospheric neutrinos. The method of event selection relies on the application of a series of cuts which are tailored to the response of the telescope to nearly vertically upward moving muons [10]. The applied cuts select muons with $-1<\cos (\theta)<-0.75$ and result in a detection area of about $1800 \mathrm{~m}^{2}$ for vertically upward going muons. The energy threshold for this analysis is $E_{\mathrm{thr}} \sim 10 \mathrm{GeV}$ i.e. significantly lower then for the analysis described in section $2.1\left(E_{\mathrm{thr}} \sim 15-20 \mathrm{GeV}\right)$. Therefore the effect of oscillations is stronger visible. We expect a muon event suppression of (25-30)\% due to neutrino oscillations assuming $\delta m^{2}=2.5 \cdot 10^{-3} \mathrm{eV}^{2}$ with full mixing, $\theta_{m} \approx \pi / 4$.

From 1038 days of effective data taking between April 1998 and February 2003, 48 events with $-1<\cos (\theta)<-0.75$ have been selected as clear neutrino events, compared to 56.6 events expected from atmospheric neutrinos in case of oscillations and 73.1 events without oscillations. The angular distribution of these events as well as the MC - predicted distributions are shown in Fig. 2. For the MC simulations we used the Bartol-96 atmospheric neutrino flux [11] without (dashed curve) and with (solid curve) oscillations. Within statistical uncertainties the experimental angular distribution is consistent with the prediction including neutrino oscillations.

Regarding the 48 detected events as being induced by atmospheric neutrinos, one can derive an upper limit on the additional flux of muons from the center of the Earth due to annihilation of neutralinos - the favored candidate

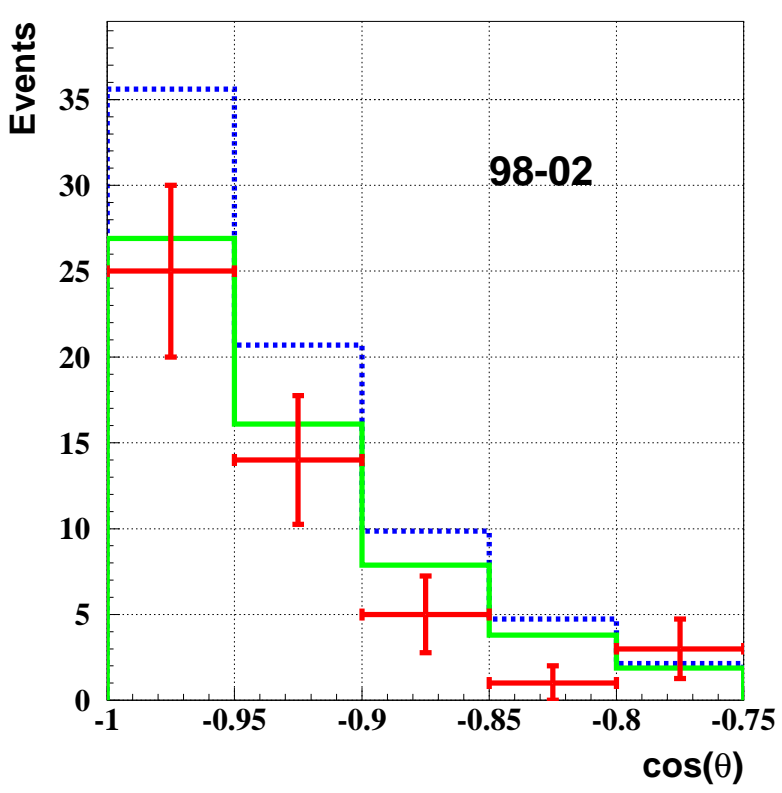

Fig. 2. Angular distributions of selected neutrino candidates as well as expected distributions in a case with and without oscillations (solid and dashed curves respectively).

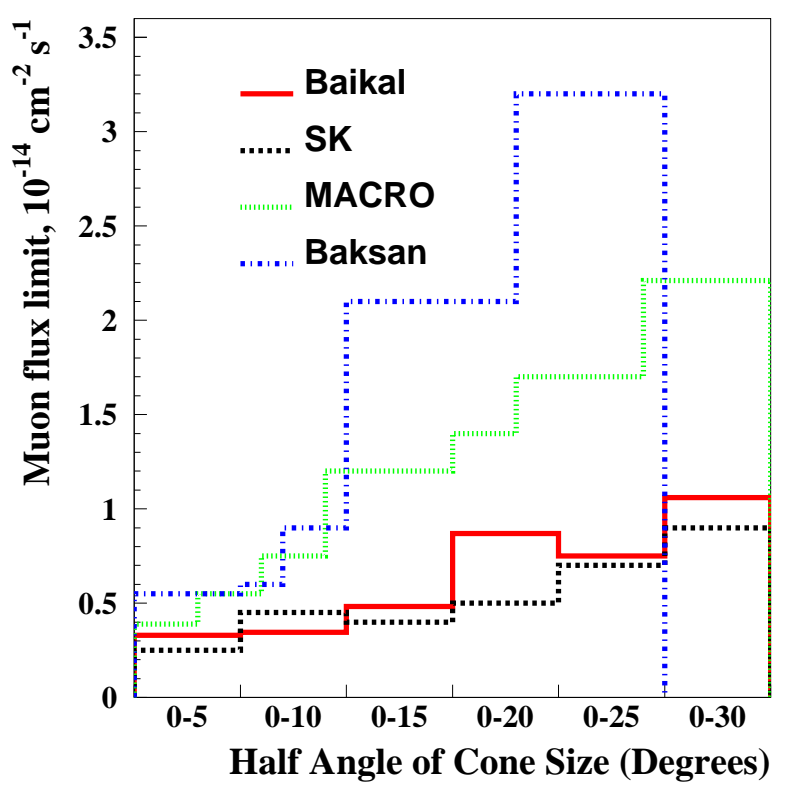

Fig. 3. Limits on the excess of muon flux from the center of the Earth versus half-cone of the search angle.

for cold dark matter. The $90 \%$ C.L. muon flux limits for six cones around the opposite zenith obtained with NT200 $\left(E_{\text {thr }}>10 \mathrm{GeV}\right)$ in $1998-2002$ are shown in Fig. 3. It was shown [12-14] that the size of a cone which contains $90 \%$ of signal strongly depends on neutralino mass. $90 \%$ C.L. flux limits are calculated as a function of neutralino mass using cones which collect $90 \%$ of the expected signal and are corrected for the $90 \%$ collection efficiency due to cone size. Also a correction is applied for each neutralino mass to translate from $10 \mathrm{GeV}$ to $1 \mathrm{GeV}$ threshold (thus modifying the results as presented earlier for $10 \mathrm{GeV}$ threshold [16]). These limits are shown in Fig. 4. Also shown in Fig. 3, and Fig. 4 


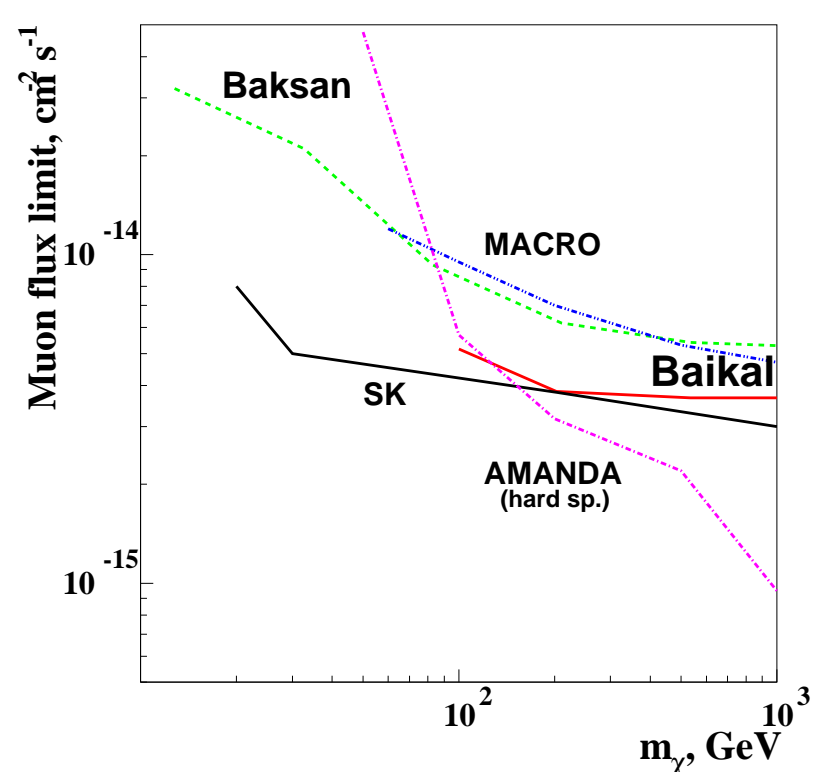

Fig. 4. Limits on the excess of muon flux from the center of the Earth as a function of neutralino mass.

are limits obtained by Baksan [12], MACRO [13], SuperKamiokande [14] and AMANDA (from the hard neutralino annihilation channels) [15].

\subsection{A search for fast magnetic monopoles}

Fast magnetic monopoles with Dirac charge $g=68.5 e$ are interesting objects to search for with deep underwater neutrino telescopes. The intensity of monopole Cherenkov radiation is $\approx 8300$ times higher than that of muons. Optical modules of the Baikal experiment can detect such an object from a distance up to hundred meters. The processing chain for fast monopoles starts with the selection of events with a high multiplicity of hit channels: $N_{\text {hit }}>30$. In order to reduce the background from downward atmospheric muons we restrict ourself to monopoles coming from the lower hemisphere. For an upward going particle the times of hit channels increase with rising $z$-coordinates from bottom to top of the detector. To suppress downward moving particles, a cut on the value of the time- $z$-correlation, $C_{t z}$, is applied.

Within 994 days of live time using in this analysis, about $3 \cdot 10^{8}$ events with $N_{\text {hit }}>4$ have been recorded, with 20943 of them satisfying cut $0\left(N_{h i t}>30\right.$ and $\left.C_{t z}>0\right)$. For further background suppression (see [17] for details of the analysis) we use additional cuts, which essentially reject muon events and at the same time only slightly reduce the effective area for relativistic monopoles.

The upper limit on a flux of magnetic monopoles with $\beta=1$ is $4.6 \cdot 10^{-17} \mathrm{~cm}^{-2} \mathrm{~s}^{-1} \mathrm{sr}^{-1}$. In Fig. 5 we compare our upper limit for an isotropic flux of fast monopoles obtained with the Baikal neutrino telescope to the limits from the underground experiments Ohya [19] and MACRO[20] and to the limit reported for the underice detector AMANDA B10 [21] and preliminary limit for AMANDA II [22].

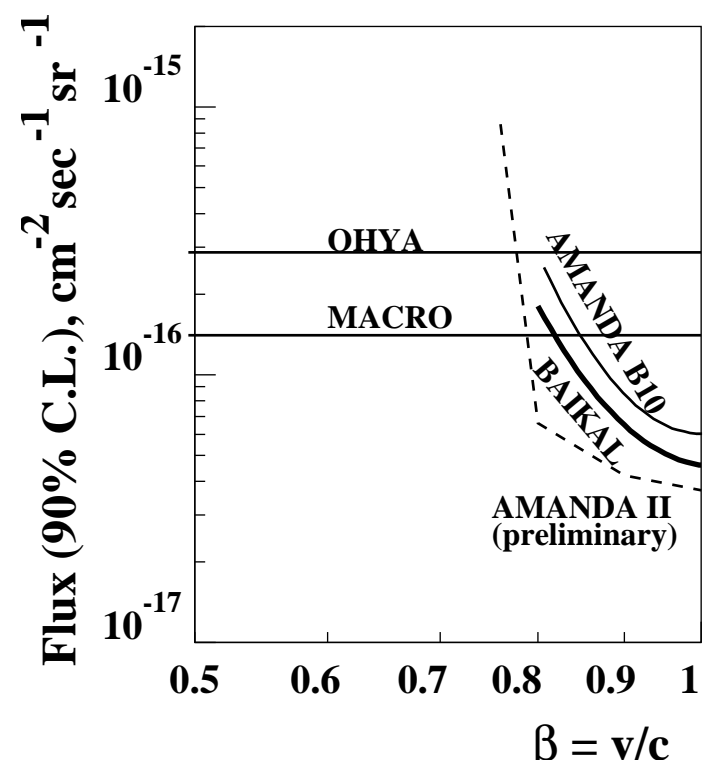

Fig. 5. Upper limits on the flux of fast monopoles obtained in this analysis (Baikal) and in other experiments.

\subsection{A search for extraterrestrial high-energy neutrinos}

The BAIKAL survey for high energy neutrinos searches for bright cascades produced at the neutrino interaction vertex in a large volume around the neutrino telescope [3]. We select events with high multiplicity of hit channels $N_{\text {hit }}$, corresponding to bright cascades. To separate highenergy neutrino events from background events a cut to select events with upward moving light signals has been developed. We define for each event $t_{\min }=\min \left(t_{i}-t_{j}\right)$, where $t_{i}, t_{j}$ are the arrival times at channels $i, j$ on each string, and the minimum over all strings is calculated. Positive and negative values of $t_{\min }$ correspond to upward and downward propagation of light, respectively.

Within the 1038 days of the detector live time between April 1998 and February 2003, $3.45 \times 10^{8}$ events with $N_{\text {hit }} \geq 4$ have been recorded. For this analysis we used 22597 events with hit channel multiplicity $N_{\text {hit }}>15$ and $t_{\min }>-10 \mathrm{~ns}$. We conclude that data are consistent with simulated background for both $t_{\min }$ and $N_{\text {hit }}$ distributions. No statistically significant excess above the background from atmospheric muons has been observed. To maximize the sensitivity to a neutrino signal we introduce a cut in the $\left(t_{\min }, N_{\text {hit }}\right)$ phase space.

Since no events have been observed which pass the final cuts upper limits on the diffuse flux of extraterrestrial neutrinos are calculated. For a $90 \%$ confidence level an upper limit on the number of signal events of $n_{90 \%}=2.5$ is obtained assuming an uncertainty in signal detection of $24 \%$ and a background of zero events.

A model of astrophysical neutrino sources, for which the total number of expected events, $N_{m}$, is larger than $n_{90 \%}$, is ruled out at $90 \% \mathrm{CL}$. Table 1 represents event rates and model rejection factors (MRF) $n_{90 \%} / N_{m}$ for models of astrophysical neutrino sources obtained from our search, as 

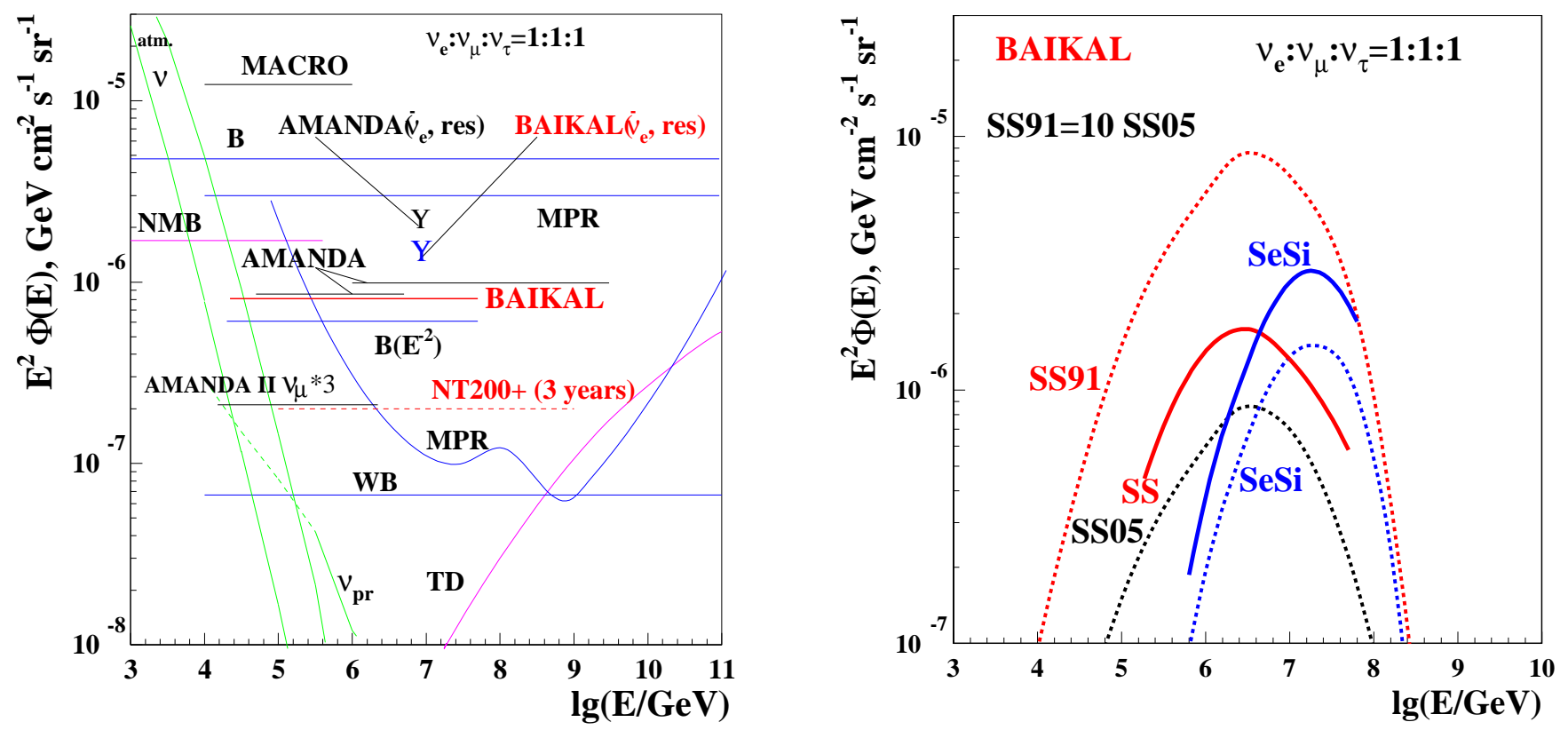

Fig. 6. Left panel: all-flavor neutrino flux predictions in different models of neutrino sources compared to experimental upper limits to $E^{-2}$ fluxes obtained by this analysis and other experiments (see text). Also shown is the sensitivity expected for 3 live years of the new telescope NT200+ [5,45]. Right panel: Baikal experimental limits compared to two model predictions. Dotted curves: predictions from model SS [26], SeSi [32] and SS05 [27]. Full curves: obtained experimental upper limits to spectra of the same shape. Model SS is excluded (MRF=0.25), model SeSi is not $(\mathrm{MRF}=2.12)$.

Table 1

Expected number of events $\mathrm{N}_{m}$ and model rejection factors for model of astrophysical neutrino sources.

\begin{tabular}{lcc|c}
\hline & \multicolumn{2}{c|}{ BAIKAL } & AMANDA $[23-25]$ \\
\hline Model & $\nu_{e}+\nu_{\mu}+\nu_{\tau}$ & $n_{90 \%} / N_{\mathrm{m}}$ & $n_{90 \%} / N_{\mathrm{m}}$ \\
\hline $10^{-6} \times E^{-2}$ & 3.08 & 0.81 & 0.22 \\
SS Quasar [26] & 10.00 & 0.25 & 0.21 \\
SS05 Quasar [27] & 1.00 & 2.5 & 1.6 \\
SP u [28] & 40.18 & 0.062 & 0.054 \\
SP l [28] & 6.75 & 0.37 & 0.28 \\
P $p \gamma[29]$ & 2.19 & 1.14 & 1.99 \\
M $p p+p \gamma[30]$ & 0.86 & 2.86 & 1.19 \\
MPR [31] & 0.63 & 4.0 & 2.0 \\
SeSi [32] & 1.18 & 2.12 & - \\
\hline
\end{tabular}

well as model rejection factors obtained recently by the AMANDA collaboration [23-25].

For an $E^{-2}$ behaviour of the neutrino spectrum and a flavor ratio $\nu_{e}: \nu_{\mu}: \nu_{\tau}=1: 1: 1$, the $90 \%$ C.L. upper limit on the neutrino flux of all flavors obtained with the Baikal neutrino telescope NT200 (1038 days) is:

$E^{2} \Phi<8.1 \times 10^{-7} \mathrm{~cm}^{-2} \mathrm{~s}^{-1} \mathrm{sr}^{-1} \mathrm{GeV}$.

For the resonant process with the resonant neutrino energy $E_{0}=6.3 \times 10^{6} \mathrm{GeV}$ the model-independent limit on $\overline{\nu_{e}}$ is:

$\Phi_{\overline{\nu_{e}}}<3.3 \times 10^{-20} \mathrm{~cm}^{-2} \mathrm{~s}^{-1} \mathrm{sr}^{-1} \mathrm{GeV}^{-1}$.
Fig. 6 (left panel) shows our upper limit on the all flavor $E^{-2}$ diffuse flux (1) as well as the model independent limit on the resonant $\bar{\nu}_{e}$ flux (diamond) (2). Also shown are the limits obtained by AMANDA [23-25] and MACRO [33], theoretical bounds obtained by Berezinsky (model independent (B) [34] and for an $E^{-2}$ shape of the neutrino spectrum $\left(\mathrm{B}\left(E^{-2}\right)\right)[35]$, by Waxman and Bahcall (WB) [36], by Mannheim et al.(MPR) [31], predictions for neutrino fluxes from topological defects (TD) [32], prediction on diffuse flux from AGNs according to Nellen et al. (NMB) [37], as well as the atmospheric conventional neutrino fluxes [38] from horizontal and vertical directions ( $(\nu)$ upper and lower curves, respectively) and atmospheric prompt neutrino fluxes $\left(\nu_{p r}\right)$ obtained by Volkova et al. [39]. The right panel of Fig. 6 shows our upper limits (solid curves) on diffuse fluxes from AGNs shaped according to the model of Stecker and Salamon (SS, SS05) [26,27] and of Semikoz and Sigl (SeSi) [32], according to Table 1.

\section{A prototype device for acoustic neutrino detection in Lake Baikal}

Back in 1957, G.A. Askaryan has shown that a highenergy particle cascade in water, besides the Cherenkov radiation, should also produce an acoustic signal of bipolar form[40]. The absorption length for acoustic waves with a frequency about $30 \mathrm{kHz}$ (the peak frequency of acoustic signals from a shower) in sea water is at least an order of magnitude larger than that of Cherenkov radiation, in 


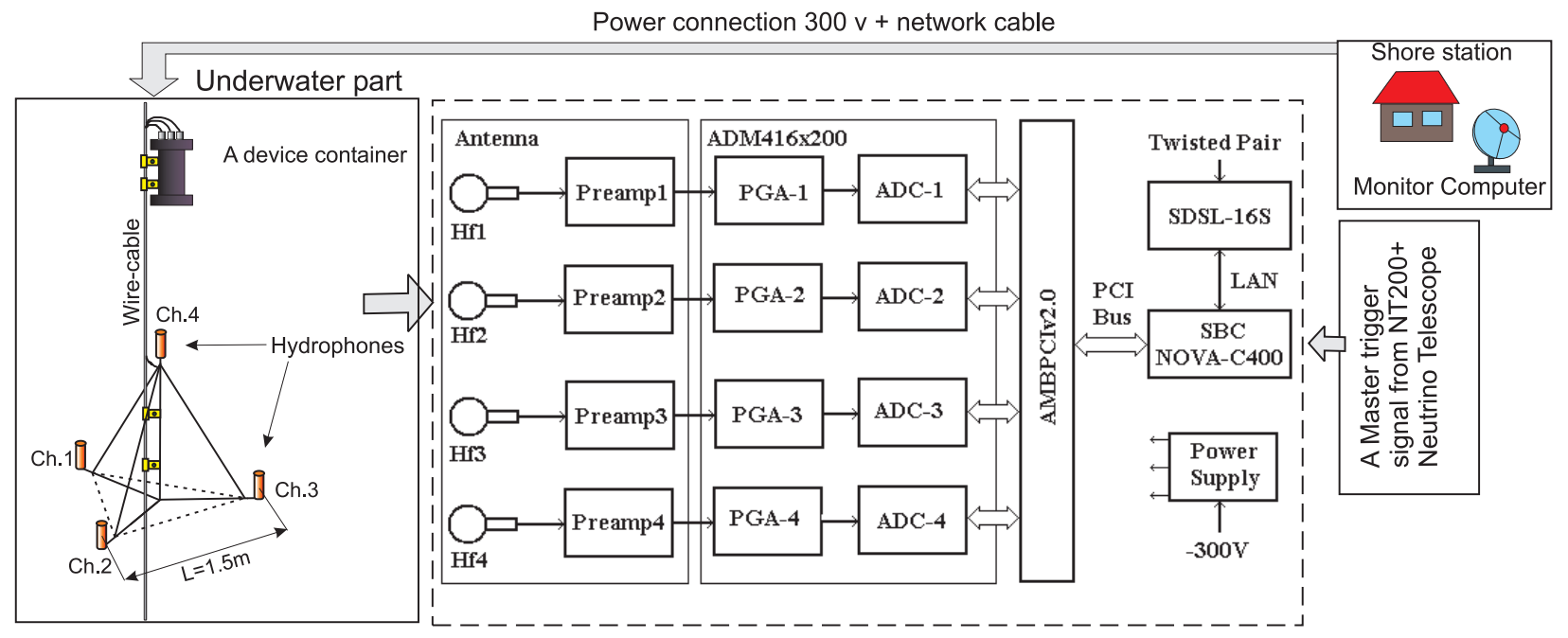

Fig. 7. Schematic view of the underwater 4-channel digital device for detection of acoustic signals from high energy neutrinos.

the fresh Baikal water this ratio is even close to 100 [41]. Therefore acoustic pulses can be detected from considerably larger distances than Cherenkov radiation, and the acoustic method appears to be attractive for the detection of ultra high-energy neutrinos. However, the technology of acoustic detection in high-energy physics is much worse developed than optical methods. Since several years, an increasing number of feasibility studies on acoustic particle detection are performed [42].

In order to test the possibility of acoustic detection of high-energy neutrinos in Lake Baikal, the Baikal collaboration started with an in-situ study of acoustic noise which constitutes the background for the acoustic neutrino detection in the lake. We have performed a series of hydroacoustic measurements in Lake Baikal in order to investigate the background properties [43]. It turned out that at stationary and homogeneous meteorological conditions the integral noise power in the frequency range $20-50 \mathrm{kHz}$ can reach levels as low as about $1 \mathrm{mPa}$. At the same time, short acoustic pulses with different amplitudes and shapes including bipolar ones have been observed. The latter should be considered as a background for acoustic neutrino detection.

Extraction of small signals from background requires an antenna consisting of a set of hydrophones. We have constructed a digital hydro-acoustic device with four input channels shown in Fig. 7 (see [44]). The module was designed for common operation with the Baikal Neutrino Telescope NT200+ and has been installed in April 2006 at one of the moorings of NT200+.

Fig. 8 presents an example of a bipolar pulse, detected by this device. It was shown that most of pulses are located in the vicinity of the horizontal plane. Sources of pulses coming from just below horizon are likely located in the nearsurface zone. They appear to come from below horizon due to refraction which is caused by the growth in sound velocity with depth. From the region $\pm 45^{\circ}$ around the opposite zenith, no event with bipolar pulse form has been observed.

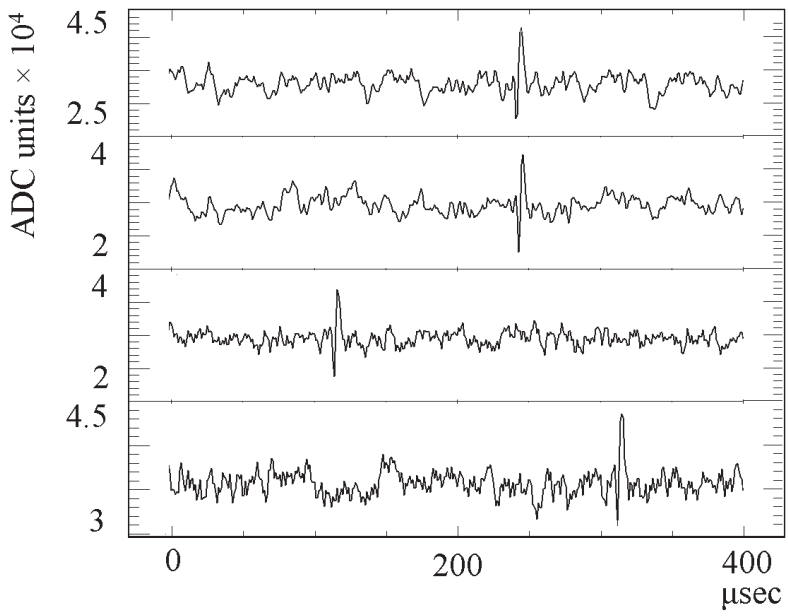

Fig. 8. Sample of detected bipolar pulse (channel 1-4).

The results of the experiment have demonstrated the feasibility of the proposed acoustic pulse detection technique in searching signals from cascade showers. Although the Baikal water temperature is close to the temperature of its maximal density, the absence of strong acoustic noise sources in the lake's deep zone, and the very low absorption of sound in freshwater may result in neutrino detection in Lake Baikal with a threshold as low as $10^{18}-10^{19} \mathrm{eV}$. This motivates further activities towards a large-scale acoustic neutrino detector in Lake Baikal.

\section{Towards a km3 detector in Lake Baikal}

The construction of NT200+ is a first step towards a km3-scale Baikal neutrino telescope. Such a detector could be made of building blocks similar to NT200+, but with NT200 replaced by a single string, still allowing separation of high-energy neutrino induced cascades from background. It will contain a total of $1300-1700$ optical modules (OM)s, arranged at 90-100 strings with 12-16 OMs each, and a length of $300-350 \mathrm{~m}$. Interstring distance will be $\approx 100 \mathrm{~m}$. 
The effective volume for detection of cascades with energy above $100 \mathrm{TeV}$ is $0.5-0.8 \mathrm{~km}^{3}$.

The existing NT200+ allows to verify all key elements and design principles of the km3 (Gigaton-Volume) Baikal telescope. The next step of the ongoing $\mathrm{km} 3$-telescope research and development work (R\&D) was the installation of a "new technology" prototype string as part of NT200+ in spring 2008. This string consists of 6 optical modules with 2 PM R8055 (13" Hamamatsu) and 4 PM XP1807 (12" Photonis), as sketched in Fig. 9. The distance between OMs on the string is $10 \mathrm{~m}$. Tubes of $4 \mathrm{OMs}$ are mounted with downward looking photocathode. The two bottom OMs contain a PM with upward looking photocathode. Dynode outputs of all 6 PMs are connected through underwater coaxial cables with an 8-channel $200 \mathrm{MHz}$ FADC board located in a separate glass sphere. Two additional FADC channels are used for pulse form measuring from 2 additional low-gain channels of two upper PMs. Data from the FADC are transmitted through an Ethernet line to an underwater micro$\mathrm{PC}$ for on-line analysis and data compression. Communication between PC-unit and the underwater control center of $\mathrm{NT} 200+$ is provided by xDSL modems trough 2-wire line of about $1 \mathrm{~km}$ length. A string trigger is formed by FADC unit under the conditions of 2 or 3 -fold coincidences of PM pulses. Time synchronization of the prototype string and telescope data acquisition systems is the same as for the outer strings of the NT200+ detector: the string trigger do generate a request that is transmitted to the detector control center were a common for all strings acknowledgment signal is formed. Time and amplitude calibration is provided by a string LED flasher. The LED flasher is located in a separate glass sphere near the FADC and PC units. Light pulses from the flasher are transmitted to each OM through the optical fibers with calibrated length. These LED flashers allow to obtain the time offsets of the channels, and provide the possibility of single electron spectrum monitoring for all PMs of the string. Control and monitoring of the OMs and operation of the LED flashers is provided by a PC unit through RS-485 underwater bus. The main slow control functions are the regulation of PM high voltage, the installation of LED flasher intensity and pulse delay, and the measuring of the rate of the PM pulses. First in-situ tests of the prototype string with underwater laser and LED flashers show the good performance of all string elements.

\section{Selected results of site studies}

Processes of horizontal and vertical water exchanges are of great importance for preservation of the Baikal ecosystem. They supply all layers of the $1640 \mathrm{~m}$ deep lake with oxygen, redistribute soluted and suspended matter and prevent accumulation of toxic substances. At the same time, it is also interesting to study hydrophysical processes in Lake Baikal from the physical point of view. Due to specific geographical, meteorological and other factors a number of

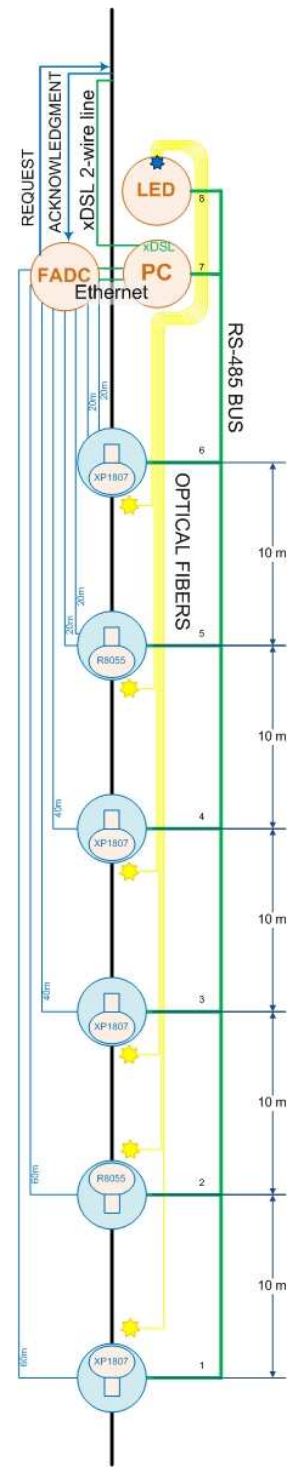

Fig. 9. Prototype string of the Gigaton-Volume Baikal neutrino telescope.

different phenomena with space-time scales ranging from a fraction of millimeters and seconds up to several hundreds of kilometers and several years take place in the Lake. Longterm monitoring of different parameters of Baikal water as the working medium of the Baikal neutrino telescope as well studying of deep-water renewal [47] and its influence on both biological and biogeochemical processes is an important part of the Baikal Neutrino Experiment. For example, the counting rate behaviour of the PMs of the neutrino telescope have been used to trace the seasonal behaviour of bio-luminenscence, and also to follow short term flows of water loaded with bio-luminescent matter [48].

A substantial factor, which influences hydrophysical as well hydrobiological and hydrochemical processes in Lake Baikal is its temperature conditions; and at the same time the water temperature is a very efficient tracer to study hydrophysical phenomena in a natural basin. We started 
regular long-term high-precision measurements of temperature in March 1999 in cooperation with the Swiss Federal Institute of Environmental Science and Technology (EAWAG). We have been using more than 50 temperature loggers TR-1000, TR-1050 and other types manufactured by the RBR company. They have been distributed between the lake bottom and $15 \mathrm{~m}$ water depth at 3 moorings in the vicinity of $\mathrm{NT} 200+$. Around the year all instruments measure the temperature each 10 min with a resolution better than $0.002^{\circ} \mathrm{C}$.

Fig.10 shows an example of temporal changes of the temperature in the depth interval 19-182 $\mathrm{m}$ for the years 20002001. A lot of hydrophysical phenomena are clearly seen from the picture in detail: spring warming and autumn cooling of water in the layer which lead to a vertical mixing of water due to free convection and homothermy of $300 \mathrm{~m}$ layer twice per year, different types of inner waves, upwellings and so on. In summer during a long time the water temperature below $77 \mathrm{~m}$ practically coincides with the temperature of maximal density at the corresponding depths [49] and the vertical coefficient of the thermodiffusion can sometimes decrease up to $0.006 \mathrm{~m}^{2} / \mathrm{s}$, it is close to the value of the molecular thermodiffusion coefficient of $-0.002 \mathrm{~m}^{2} / \mathrm{s}$.

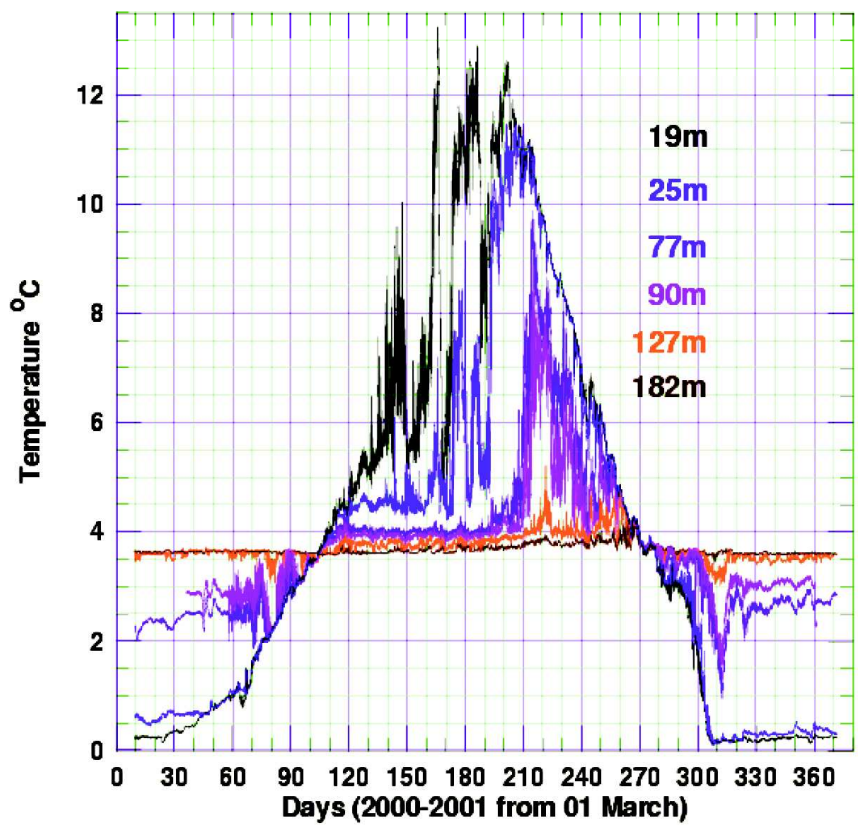

Fig. 10. Dependence of temperature on time in upper layer of Lake Baikal during one year.

The water temperature below $300 \mathrm{~m}$ slowly decreases with depth and density of water is perfectly stratified all around the year. But almost simultaneous temperature variations down to $1000 \mathrm{~m}$ depth are observed. The structure of the variations at different depths correlate, although the amplitude decreases with depth. These disturbances have a maximum magnitude in the period close to the autumn homothermy and are connected with intrusion of water from upper layers. The average temperature at the depth of $500 \mathrm{~m}$ is $3.52^{\circ} \mathrm{C}$ and the maximum observed amplitude of temperature disturbances at this depth was $0.15^{\circ} \mathrm{C}$. At a depth of $996 \mathrm{~m}$ these values were $3.40^{\circ} \mathrm{C}$ and $0.03^{\circ} \mathrm{C}$ respectively. Significant disturbances of smaller amplitude, when the temperature increases and decreases compare with the typical value, have been observed practically all around the year, including the ice period. Two basic mechanisms resulting in the water displacement over a large range of depths were suggested: thermal bars [51] and fronts [52]. However, data never showed cases when such vertical disturbances reach down to the bottom. So, they can not be responsible for huge cold water intrusions in the nearbottom zone which have been regularly observed around the time of convective surface mixing in June and December/January and have a typical volume of about $10 \mathrm{~km}^{3}$. One of the largest intrusions was observed in June 2000 when the temperature at depth $1362 \mathrm{~m}$ suddenly decreased nearly by $0.1^{\circ} \mathrm{C}$ and returned to its initial value only after 6 months (see Fig. 11). Such intrusions play a crucial role in oxygenation of deepest layers of the lake and lead to an efficient recycling of the nutrients from the deep water. But up to now, a convincing explanation of this phenomenon is missing. The first time direct observations which prove that these advective intrusions are caused by coastal downwelling and subsequent thermobaric instability along the steep lake shores was made using loggers distributed on our 3 moorings including one located at distance $1 \mathrm{~km}$ from shore [53].

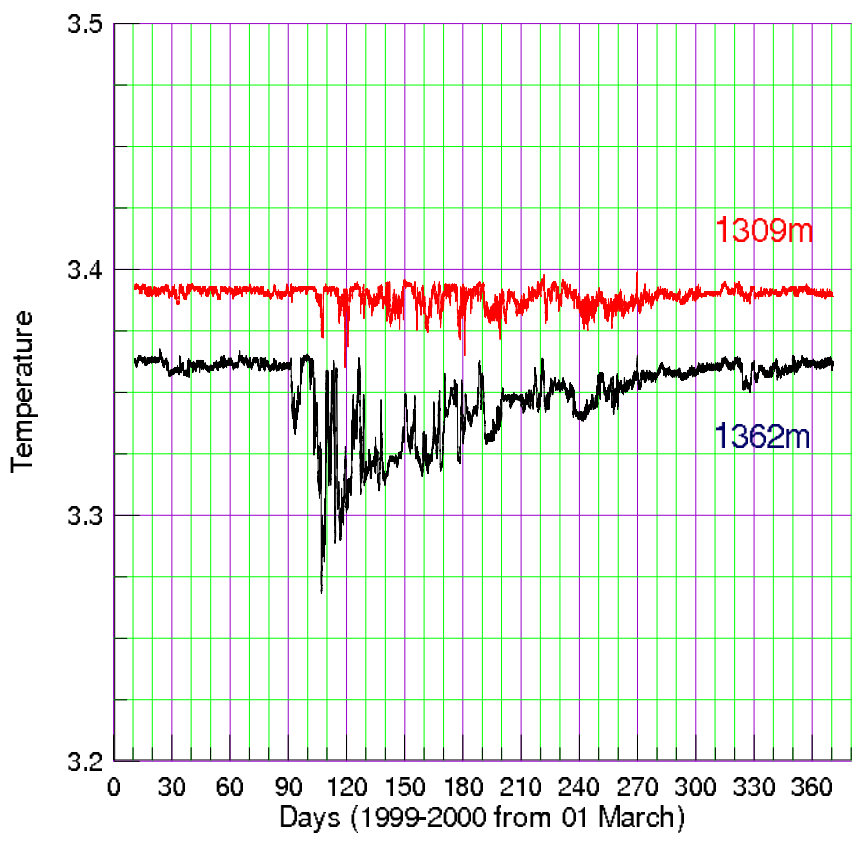

Fig. 11. Temperature at $4 \mathrm{~m}$ and $57 \mathrm{~m}$ from bottom in 1999-2000 year.

\section{Conclusion}

The Baikal neutrino telescope NT200 is taking data since April 1998. The upper limit obtained for a dif- 
fuse $\left(\nu_{e}+\nu_{\mu}+\nu_{\tau}\right)$ flux with $E^{-2}$ shape is $E^{2} \Phi=$ $8.1 \times 10^{-7} \mathrm{~cm}^{-2} \mathrm{~s}^{-1} \mathrm{sr}^{-1} \mathrm{GeV}$. The limits on the flux of fast magnetic monopoles and on an additional muon flux induced by WIMP annihilation at the center of the Earth belong to the most stringent limits existing to date. The limit on a $\overline{\nu_{e}}$ flux at the resonant energy $6.3 \times 10^{6} \mathrm{GeV}$ is presently the most stringent.

To extend the search for diffuse extraterrestrial neutrinos with higher sensitivity, NT200 was significantly upgraded to NT200+, a detector with about 5 Mton enclosed volume, which takes data since April 2005 [5,45]. The threeyear sensitivity of NT200+ to the all-flavor neutrino flux is approximately $2 \times 10^{-7} \mathrm{~cm}^{-2} \mathrm{~s}^{-1} \mathrm{sr}^{-1} \mathrm{GeV}$ for $E>10^{2} \mathrm{TeV}$ (shown in Fig. 6).

For a km3-scale detector in Lake Baikal, R\&D-activities are in progress. The NT200+ detector is, beyond its better physics sensitivity, used as an ideal testbed for critical new components. Modernization of the NT200+ DAQ allowed to install a prototype FADC PM readout. A prototype new technology string with six large area hemispherical PMs (4 Photonis XP1807/12" and 2 Hamamatsu R8055/13"), was installed in spring 2008 and a km3-detector Technical Design Report is planned for fall 2008.

\section{Acknowledgements}

This work was supported by the Russian Ministry of Education and Science, the German Ministry of Education and Research, REC-17 BAIKAL and the Russian Fund of Basic Research (grants 07-02-00791, 08-02-00198, 08-02-10001), and by the Grant of President of Russia NSh-4580.2006.2. and by NATO-Grant NIG-9811707(2005).

\section{References}

[1] I. Belolaptikov et al. Astropart. Phys. 7 (1997) 263.

[2] V. Aynutdinov et al. Nucl. Phys. (Proc. Suppl.) B143 (2005) 335 .

[3] V. Aynutdinov et al. Astropart. Phys. 25 (2006) 140.

[4] V. Aynutdinov et al. in: Proc. of V Int. Conf. on Non-Accelerator New Physics June 7-10 (2005) Dubna Russia.

[5] V. Aynutdinov et al. NIM A567 (2006) 433.

[6] V. Aynutdinov et al. in: Proc. 30th ICRC (icrc1084), Merida, 2007; arXiv.org: astro-ph/0710.3063.

[7] V. Aynutdinov et al. in: Proc. 30th ICRC (icrc1088), Merida, 2007; arXiv.org: astro-ph/0710.3064.

[8] V. Balkanov et al. Astropart. Phys. 12 (1999) 75.

[9] V. Aynutdinov et al. Int. J. Mod. Phys. B20 (2005) 6932.

[10] V. Balkanov et al. Nucl.Phys. (Proc.Suppl.) B91 (2001) 438.

[11] V. Agrawal, T. Gaisser, P. Lipari \& T. Stanev Phys. Rev. D53 (1996) 1314.

[12] M. Boliev et al. Nucl. Phys. (Proc. Suppl.) 48 (1996) 83; O. Suvorova arXiv.org: hep-ph/9911415 (1999).

[13] M. Ambrosio et al. Phys. Rev. D60 (1999) 082002.

[14] S. Desai et al. Phys. Rev. D70 (2004) 083523; erratum ibid D70 (2004) 109901.

[15] J. Ahrens et al. arXiv.org: astro-ph/0509330 (2005).

[16] K. Antipin et al. in: Proc. of the First Workshop on Exotic Physics with Neutrino Telescopes, Uppsala, Sweden, Sept. 20-22 (2006) 34 .
[17] K. Antipin et al. in: Proc. of the First Workshop on Exotic Physics with Neutrino Telescopes, Uppsala, Sweden, Sept. 20-22 (2006) 80.

[18] I. Belolaptikov et. al. [Baikal collaboration] in: 26th ICRC, Salt Lake City, V.2 (1999) 340.

[19] S. Orito et. al. Phys.Rev.Lett. 66 (1991) 1951.

[20] M. Ambrosio et. al. [MACRO collaboration] arXiv.org: hepex/02007020 (2002).

[21] P. Niessen, C. Spiering [AMANDA collaboration] in: 27th ICRC, Hamburg, V.4 (2001) 1496.

[22] H. Wissing et al. Ice Cube Collaboration, in: Proc. 30th ICRC, Merida, 2007.

[23] M. Ackermann et al. Astropart. Phys. 22 (2005) 127; Astropart. Phys. 22 (2005) 339.

[24] M. Ackermann et al. Astropart. Phys. 22 (2005) 339.

[25] M. Ackermann et al. Phys. Rev. D76 (2007) 042008.

[26] F. Stecker and M. Salamon Space Sci. Rev. 75 (1996) 341.

[27] F. Stecker Phys. Rev. D72 (2005) 107301.

[28] A. Szabo and R. Protheroe in: Proc. High Energy Neutrino Astrophysics, ed. V.J. Stenger et al., Honolulu, Hawaii (1992).

[29] R. Protheroe arXiv.org:astro-ph /9612213.

[30] K. Mannheim Astropart. Phys. 3 (1995) 295.

[31] K. Mannheim, R. Protheroe and J. Rachen Phys. Rev. D63 (2001) 023003.

[32] D. Semikoz and G. Sigl, arXiv.org:hep-ph/0309328.

[33] M. Ambrosio et al. Nucl. Phys. (Proc. Suppl.) B110 (2002) 519.

[34] V. Berezinsky et al. in: Astrophysics of Cosmic Rays, Elsevier Science, North-Holland 1990.

[35] V. Berezinsky arXiv.org: astro-ph/0505220 (2005).

[36] E. Waxman and J. Bahcall Phys. Rev. D59 (1999) 023002.

[37] L. Nellen, K. Mannheim and P. Biermann Phys. Rev. D47 (1993) 5270 .

[38] L. Volkova Yad. Fiz. 31 (1980) 1510.

[39] L. Volkova and G. Zatsepin Phys. Lett. B462 (1999) 211.

[40] G. A. Askaryan. Atomnaya Energiya. 3, N.8:152, 1957.

[41] C. S. Clay and H. Medwin. Acoustical Oceanography (Wiley, New York), 1977.

[42] Proc. of Int. Workshop on Acoustic and Radio EeV Neutrino Dection Activities (ARENA2005), (World Sientific, Singapore), 2006, 298 p., Editors R. Nahnhauer and S. Böser.

[43] V. Aynutdinov et al., Int. J. Mod. Phys. A21S1 (2006) 117; V. Aynutdinov et al. Acoust. Phys. 52 N5 (2006) 495.

[44] V. Aynutdinov et al. in: Proc. 30th ICRC (icrc0639), Merida, 2007; arXiv.org: astro-ph/0710.3113.

[45] V. Aynutdinov et al. in: Proc. 29th Int. Cosmic Ray Conf. August 3-10 Pune India (2005); arXiv.org: astro-ph /0507715.

[46] R. Bagduev et al., NIM A420 (1999) 138.

[47] Weiss R.F., Carmac E.C., Koropalov V.M. Deep-water renewal and biological production in Lake Baikal // Nature. 1991. V.349. P. 665-669.

[48] Ch.Spiering et al., The Baikal Deep Underwater Neutrino Experiment: Results, Status, Future Prog.Part.Nucl.Phys. 40 (1998) 391; astro-ph/9801044

[49] Chen C. T. and Millero F. J., Precise thermodynamic properties for natural waters covering only the limnological range // Limnol. Oceanogr. 31, 1986, P. 657-682

[50] Shimaraev M. N. and Granin N. G., Temperature stratification and the mechanisms of convection in Lake Baikal // Dokl. Akad. Nauk SSSR, 321, 1991, p. 831-835 (in Russian)

[51] Shimaraev M. N., Granin N. G. and Zhdanov A. A., Deep ventilation of Lake Baikal due to spring thermal bars // Limnol. Oceanogr. 38(5), 1993, p. 1068-1072

[52] Sherstyankin P. P., Front genesis in Lake Baikal by the materials of optical observations // Dokl. RAN 326, 1992, p. 366-370

[53] M. Schmid, N. M. Budnev, N. G. Granin et al Lake Baikal deepwater renewal mystery solved// Geophysical Research Letters. 2008. in press. 